Conference abstract PPAT19

\title{
Characterization of Biomedical Surfaces by Streaming Potential Measurements
}

\author{
T. LUXBACHER
}

Anton Paar GmbH, Graz, Austria

E-mail: thomas.luxbacher@anton-paar.com (Thomas Luxbacher)

Sci Pharm. 2010; 78: 708

doi:10.3797/scipharm.cespt.8.PPAT19

The knowledge of surface charge and the isoelectric point is important for many biomedical applications. The zeta potential, formally defined as the electrical potential at the electrokinetic plane of shear, is an important property of charged solid/liquid interfaces and a descriptor of the actual surface charge of a solid immersed in a dielectric. For macroscopic solid surfaces, the zeta potential is commonly determined by the measurement of streaming potential and streaming current. The versatility of the streaming potential method allows handling of planar surfaces, cylindrical capillaries, and packed beds of granular or fibrous materials.

Besides the evaluation of the charging behavior of solid surfaces, the zeta potential is a valuable indicator for surface stability in the presence of various liquids, and for the adsorption of proteins, polypeptides, and other biomacromolecules on biomedical and biomaterial surfaces.

In this paper the application of the streaming potential method in the field of biomedical surface characterization is presented with different case studies. 